$11,12,13$

\title{
Моделирование смачивающих фазовых переходов в тонких пленках
}

\author{
(C) П.Е. Львов ${ }^{1,2}$, В.В. Светухин ${ }^{3}$, С.В. Булярскийㄹ, А.А. Павлов ${ }^{2}$ \\ ${ }^{1}$ Ульяновский государственный университет, \\ Ульяновск, Россия \\ ${ }^{2}$ Институт нанотехнологий микроэлектроники РАН, \\ Москва, Россия \\ ${ }^{3}$ Научно-производственный комплекс „Технологический центр“, \\ Москва, Зеленоград, Россия \\ E-mail: LvovPE@sv.uven.ru
}

Поступила в Редакцию 23 апреля 2019 г.

В окончательной редакции 29 мая 2019 г.

Принята к публикации 29 мая 2019 г.

На основе гидродинамической модели проведен анализ кинетики смачивающих фазовых переходов в пленках жидкости наноразмерной толщины, находящихся на поверхности подложки. В области метастабильных состояний исследованы закономерности формирования равновесных кластеров и рассчитаны соответствующие распределения толщины пленки. В области нестабильных состояний выполнен анализ кинетики фазового перехода, приводящего к образованию кластеров. На ранней стадии происходит формирование участков в виде лунок с толщиной пленки близкой к равновесной. Слияние лунок между собой приводит к перераспределению вещества в пленке и последующему формированию кластеров. Для данного процесса рассчитана кинетика среднего размера и концентрации лунок. Для образующихся кластеров исследована кинетика среднего радиуса, средней высоты, концентрации, а также их функции распределения по радиусу и высоте.

Ключевые слова: фазовые переходы смачивания, моделирование, кинетика, тонкие пленки.

DOI: $10.21883 /$ FTT.2019.10.48270.465

\section{1. Введение}

Фазовые переходы смачивания представляют собой особый вид фазовых переходов, возникающих на границах раздела [1-4]. Существование данного вида переходов было впервые теоретически обосновано Каном [2-4], которым на основе метода функционала плотности свободной энергии была построена фазовая диаграмма, описывающая равновесие сплошных пленок и капель жидкости на плоской поверхности, а также обсуждены случаи фазовых переходов полного и частичного смачивания. Данный вид фазовых переходов наблюдается экспериментально для жидкостей на поверхности подложек [3-8]. Сходные процессы наблюдаются при распаде твердых пленок [9-10], а также на границах зерен [11]. Практическая значимость исследований в этой области обусловливается необходимостью прогнозирования поведения металлических покрытий, а также определения рабочего интервала температур активных и пассивных элементов микро- и наноэлектроники, в том числе при формировании катализаторов роста углеродных нанотрубок и графена [12,13]. Углеродные наноструктуры имеют широкие перспективы применения, что побудило разработать термодинамические модели формирования наночастиц катализаторов [12-14]. Такие модели хорошо согласуются с экспериментом при использовании формирования наночастиц катализатора из газовой фазы. В этом случае равновесие в реакторе роста нанотрубок наступает за время осуществления синтеза. Однако при плавлении пленок наноразмерной толщины, находящихся на подложке, термодинамическое равновесие не всегда достигается за время формирования катализатора. В связи с этим, необходимым является развитие моделей формирования наночастиц, описывающих кинетику данного процесса.

Кинетика фазовых переходов при плавлении наноразмерных пленок металлов близка по своему характеру к гидродинамическим моделям, которые описывают различные этапы эволюции наноразмерных пленок жидкости на поверхности подложек [1,3-5,15-18]. Расчет энергии неоднородного наноразмерного слоя жидкости обычно выполняется с помощью функционала Гинзбурга-Ландау, в котором в качестве параметра порядка используется толщина пленки $h \equiv h(x, y)$, являющаяся функцией координат [3-5]

$$
F=\int_{S}\left(V(h)+\frac{1}{2} \sigma(\nabla h)^{2}\right) d S,
$$

где $\sigma-$ поверхностное натяжение жидкости ( $\sigma=$ const $), \quad V(h) \quad$ - эффективный потенциал взаимодействия пленки с подложкой, $d S=d x d y-$ элемент поверхности подложки $S$. На основе уравнения Навье-Стокса для вязкой несжимаемой жидкости на поверхности подложки и функционала (1) может быть также получено базовое уравнение, описывающее трансформацию пленки при заданных 
термодинамических условиях [3-6]:

$$
\eta \frac{\partial h}{\partial t}=\nabla\left(\frac{h^{3}}{3} \nabla\left[\frac{\partial V}{\partial h}-\sigma \nabla^{2} h\right]\right),
$$

где $\eta-$ коэффициент вязкости жидкости, образующей пленку. Производная $\partial V / \partial h$ описывает расклинивающее давление, которое возникает в тонком слое вещества, находящемся между двумя протяженными участками однородных сред [19].

Уравнение (2) формально совпадает с уравнением Кана-Хилларда с подвижностью, зависящей от параметра порядка, которое часто используется в теории фазовых переходов первого рода (см. например, [20,21]). На основе решения уравнения (2) может быть обоснована возможность формирования на поверхности пленки уединенных кластеров [3-6,15-18]. Для данных кластеров может наблюдаться укрупнение как за счет их слияния (коагуляции), так и при реализации механизма коалесценции, связанного с ростом крупных кластеров за счет исчезновения малых [15-18]. В случае формирования небольших кластеров с высокой концентрацией существенное влияние может оказывать их подвижность, при этом сами кластеры ведут себя подобно броуновским частицам [9]. Данная особенность, по всей видимости, может быть учтена при добавлении в уравнение (2) стохастической составляющей, описывающей термические флуктуации (см. например, [22]).

Задача об установлении особенностей эволюции ансамбля дисперсных частиц, сформированных на поверхности, для различных механизмов тепло- и массопереноса решалась в работах [23-25], где была исследована кинетика среднего радиуса, средней высоты и концентрации кластеров с цилиндрической геометрией на стадии коалесценции. Также на примере цилиндрических кластеров впервые была показана взаимосвязь между их высотой и радиусом на поздней стадии [23-25].

Настоящая работа посвящена решению задачи о формировании наноразмерных кластеров в процессе трансформации сплошной жидкой пленки, находящейся при постоянной температуре ниже критической температуры смачивания. Необходимо также исследовать закономерности эволюции ансамбля кластеров на поверхности подложки и установить кинетику изменения их среднего радиуса, средней высоты, концентрации, функции распределения по размерам и др.

\section{2. Эффективный потенциал взаимодействия. Безразмерное уравнение гидродинамики тонких пленок}

Одним из основных факторов, определяющих динамику жидкой пленки, является эффективный потенциал ее взаимодействия с подложкой. Различные виды эффективного потенциала взаимодействия пленки с подлож- кой $V(h)$ рассматривались в работах [3-5,26]. Считается, что эффективный потенциал взаимодействия имеет глобальный минимум, соответствующий равновесной толщине пленки при температурах ниже критической температуры смачивания $\left(T<T_{W}\right)$, имеющей значения ниже критической температуры существования жидкости [2-4]. В этом случае возможной является трансформация пленки в систему уединенных кластеров $[5,26]$. Для температур, превышающих данное критическое значение $\left(T>T_{W}\right)$, более выгодным является состояние с однородной пленкой, и кластеры не образуются.

В области температур ниже критического значения $\left(T<T_{W}\right)$ обычно используется потенциал вида

$$
V(h)=A\left[\left(\frac{h_{0}}{h}\right)^{m}-\left(\frac{h_{0}}{h}\right)^{n}\right],
$$

где $A$ и $h_{0}-$ постоянные величины при заданной температуре. Показатели степени в формуле (3) обычно принимаются постоянными величинами равными $m=8$ и $n=2$, что соответствует потенциалу Леннард-Джонса, или $m=3$ и $n=2$. Параметр $h_{0}$ имеет величину порядка нанометра (см. например, $[5,26])$. Процесс формирования кластеров на поверхности наблюдается для пленок, имеющих толщину порядка $10 \mathrm{~nm}$ и менее [3-6].

Уравнение (2) с потенциалом вида (3) удобно привести к безразмерному виду, вводя новые переменные

$$
\begin{aligned}
& l=h_{0}\left(\frac{\sigma}{A \sigma_{0}}\right)^{1 / 2}, \tau=\frac{h_{0} A}{\eta l^{2}} t, \\
& x^{*}=\frac{x}{l}, y^{*}=\frac{y}{l}, \quad \nabla^{*}=l \nabla, h^{*}=\frac{h}{h_{0}} .
\end{aligned}
$$

Подстановка (4) в (2) и (3) позволяет представить уравнение, описывающее динамику жидкости на поверхности, посредством безразмерных переменных

$$
\frac{\partial h^{*}}{\partial \tau}=\nabla^{*}\left(\frac{h^{* 3}}{3} \nabla^{*}\left[V^{*}\left(h^{*}\right)-\sigma_{0} \nabla^{* 2} h^{*}\right]\right),
$$

где $\sigma_{0}-$ безразмерное поверхностное натяжение. В данном уравнении также использован безразмерный потенциал взаимодействия $V^{*}\left(h^{*}\right)$, производная которого определяется формулой

$$
V^{*}\left(h^{*}\right)=n\left(h^{*}\right)^{-(n+1)}-m\left(h^{*}\right)^{-(m+1)} .
$$

Зависимость эффективного потенциала взаимодействия между пленкой и подложкой $V^{*}\left(h^{*}\right)$ приведена на рис. 1. Значение $h_{e q}^{*}$ соответствует равновесному значению толщины пленки, которая определяется минимумом потенциала $V^{*}\left(h^{*}\right)$. Значение $h_{s}^{*}$ для толщины пленки соответствует точке перегиба потенциала $V^{*}\left(h^{*}\right)$ и разделяет две важные области, в которых различаются механизмы фазового перехода. Для пленок, характеризуемых средней толщиной $\bar{h}^{*} \geq h_{s}^{*}$, происходит спонтанный безактивационный фазовый переход, сходный со спинодальным распадом в бинарных растворах. В этой 


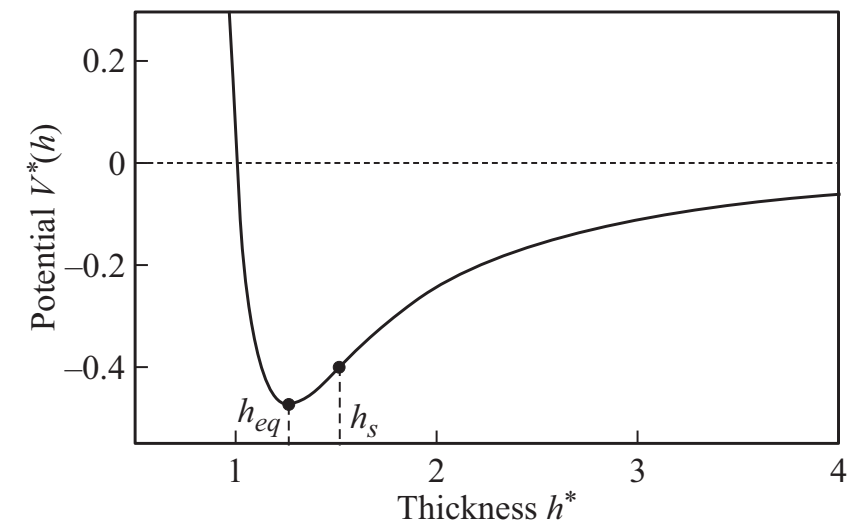

Рис. 1. Эффективный потенциал взаимодействия пленки с подложкой $V^{*}\left(h^{*}\right)$. Значения $h_{e q}^{*}=(n / m)^{1 /(m-n)}$ и $h_{s}^{*}=(n(n+1) /(m(m+1)))^{1 /(m-n)}$ соответствуют точкам минимума и перегиба потенциала.

области $V^{*^{\prime \prime}}<0$, что должно приводить к неустойчивости состояния пленки. Пользуясь аналогией с фазовыми переходами в бинарных сплавах, описываемых уравнением Кана-Хилларда, можно ожидать, что в этой области происходит формирование областей с малой толщиной пленки $\left(h^{*} \sim h_{e q}^{*}\right)$, которое протекает по спинодальному механизму [3-6,15,16]. В области метастабильных состояний $\left(V^{*^{\prime \prime}}>0, h_{e q}^{*} \leq h^{*}<h_{s}^{*}\right)$ должен наблюдаться фазовый переход, сходный с механизмом нуклеации, для реализации которого требуется преодоления барьера зародышеобразования [21-24]. Поэтому формирование зародышей для данной области возможно только за счет термических флуктуаций.

Дальнейшее рассмотрение закономерностей формирования кластеров на поверхности подложки будем проводить для потенциала (6) со значениями параметров $m=8, n=2$.

\section{3. Равновесные кластеры на поверхности тонких пленок}

Как уже обсуждалось в п. 1, в области стабильных и метастабильных состояний $\left(h_{e q}^{*} \leq h^{*}<h_{s}^{*}\right)$ могут формироваться устойчивые кластеры, для образования которых требуется преодоление барьера зародышеобразования. Барьер зародышеобразования соответствует критическим зародышам, равновесное распределение толщины пленки для которых $h(x, y)$, соответствует условию постоянства давления $p=$ const в пленке $[15,16]$ :

$$
V^{*^{\prime}}\left(h^{*}\right)-\sigma_{0} \nabla^{* 2} h^{*}=p .
$$

Здесь $p-$ константа, которая принимает значения на интервале $0<p<p_{\max }, p_{\max }=V^{*^{\prime}}\left(h_{s}^{*}\right)$. Обычно решение данной задачи выполняется для одномерного случая $[13,14]$, допускающего аналитическое интегрирование уравнения (7). В двумерном случае решение задачи может быть найдено с учетом круговой симметрии кластера, обусловленной анизотропией свойств пленки вдоль поверхности подложки. Для этого случая уравнение (7) может быть преобразовано к виду

$$
\frac{\partial^{2} h}{\partial r^{* 2}}+\frac{1}{r^{*}} \frac{\partial h}{\partial r^{*}}=\frac{\partial V^{*}}{\partial h^{*}}-\left.\frac{\partial V^{*}}{\partial h^{*}}\right|_{h_{\min }},
$$

где давление $p=\left.\frac{\partial V^{*}}{\partial h^{*}}\right|_{h_{\min }}$ определено для толщины пленки $h_{\min }$ вдали от кластера. Решение уравнения (8) проводится с учетом естественных граничных условий для выбранной симметрии кластера

$$
\frac{\partial h}{\partial r^{*}}=0, \text { при } r^{*}=0, h=h_{\min } \text { при } r^{*} \rightarrow \infty .
$$

Решение уравнения (8) с выбранными граничными условиями выполнялось с помощью метода Ньютона. Результатом решения являются равновесные зависимости толщины пленки $h(r)$ для различных значений $h_{\min }$, которые приведены на рис. 2. Из рисунка следует, что формирование кластеров происходит, когда толщина пленки вдали от кластера $h_{\text {min }}$ соответствует области стабильных и метастабильных состояний, т.е. удовлетворяет условию: $h_{e q}^{*}<h_{\mathrm{min}}^{*}<h_{s}^{*}$. В результате решения уравнения (8) (рис. 2) была также установлена зависимость толщины пленки в центре кластера $\left(h_{\max }^{*}\right)$ и вдали от него $\left(h_{\min }^{*}\right)$ как функции его линейного размера. В качестве линейного размера кластера в данном разделе был принят радиус $R_{1 / 2}$, для которой толщина пленки принимает значение $h_{1 / 2}^{*}=\left(h_{\min }^{*}+h_{\max }^{*}\right) / 2$. Результаты расчета данных зависимостей приведены на рис. 3, $a$ и $b$, из которых следует, что вблизи границы метастабильности, которая соответствует значению $h_{s}^{*}$, толщина пленки изменяется очень слабо от $h_{\min }^{*} \sim h_{s}^{*}-0$ до $h_{\max }^{*} \sim h_{s}^{*}+0$, а соответствующий линейный размер $R_{1 / 2}$

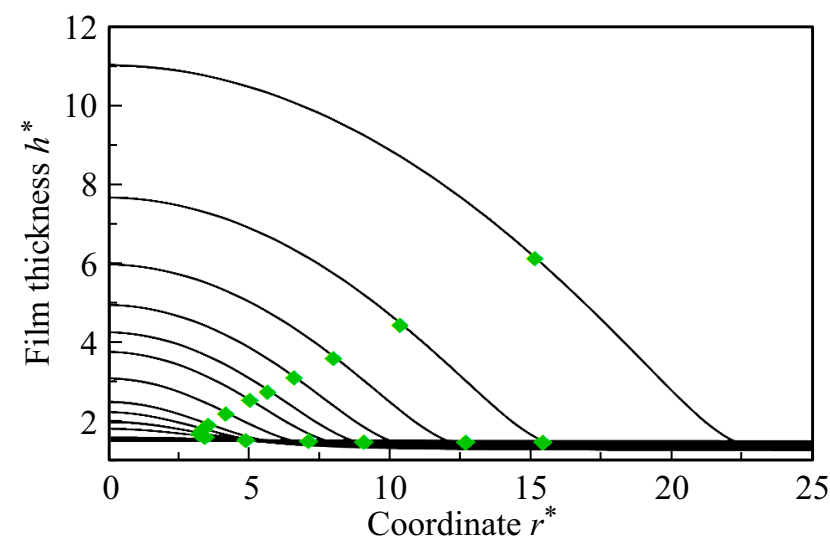

Рис. 2. Распределение пленки по толщине $h^{*}(r)$ для равновесных кластеров в ультратонких пленках $\left(\sigma_{0}=1.0\right)$, характеризуемых толщиной $h_{\min }$. Точками отмечены положения соответствующие половине высоты кластера $\left(R_{1 / 2}\right)$. Соответствие толщины $h_{\min }$ и кривой устанавливается в порядке обхода точек, соответствующих $R_{1 / 2}$ (справа налево и снизу вверх): $1.511,1.510,1.507,1.503,1.490,1.450,1.425,1.400,1.380,1.350$, $1.330,1.320,1.310,1.300,1.290,1.280$. 

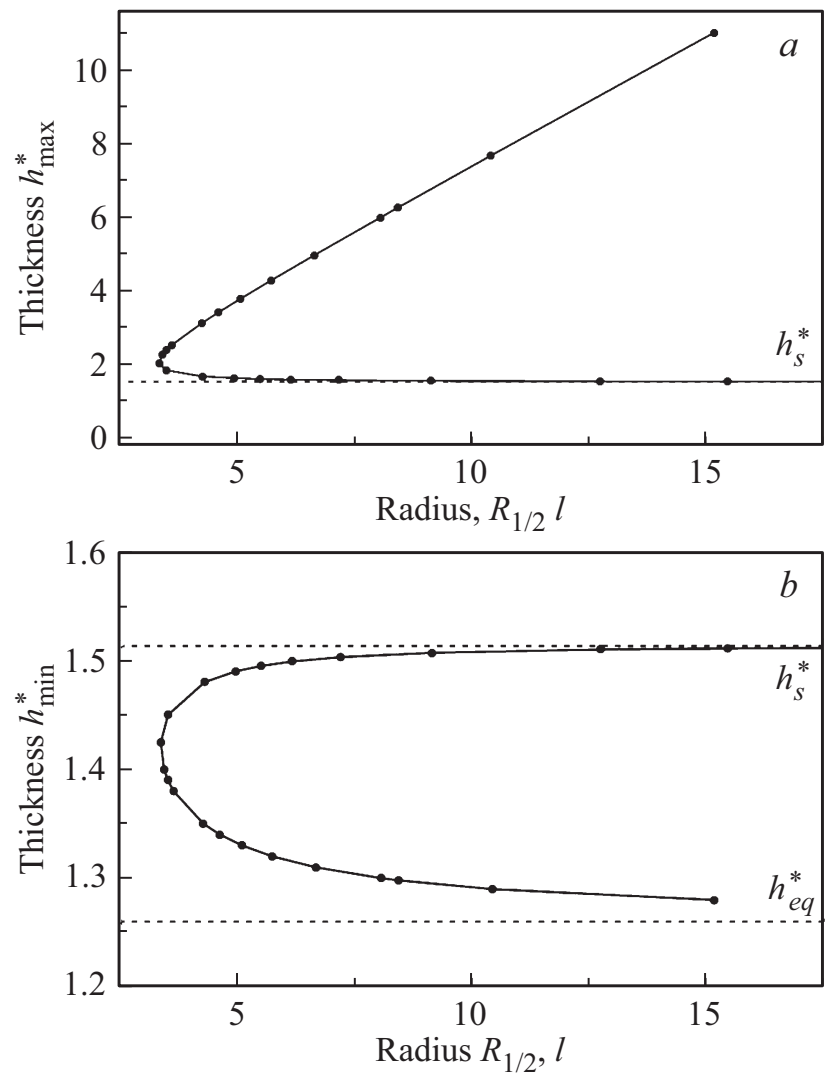

Рис. 3. Максимальная $h_{\max }$ (рис. $a$ ) и минимальная $h_{\min }$ толщина пленки (рис. $b$ ) как функции линейного размера кластера $R_{1 / 2}$, полученные по результатам расчета распределения толщины для равновесных кластеров (рис. 2).

неограниченно возрастает. В целом данная особенность аналогична образованию критических зародышей в неклассической теории нуклеации вблизи границы метастабильности [27-30]. Однако существенным различием рассматриваемой системы является отсутствие ограничения сверху по параметру порядка, что связано с формой потенциала (рис. 1). При формировании кластеров в растворах в качестве параметра порядка рассматривается величина, пропорциональная локальному составу сплава, который может принимать значения от нуля до единицы. В тонкой пленке на подложке реализуется неограниченное прямопропорциональное возрастание толщины пленки в центре равновесного кластера $h_{\max }^{*}$ с увеличением его линейного размера $R_{1 / 2}$ (см. рис. 2 и $3, a$ ). Наличие корреляции между толщиной $h_{\max }^{*}$ и линейным размером $R_{1 / 2}$ согласуется с выводами, сделанными в работах [23-25], где на примере островков с цилиндрической геометрией была показана взаимосвязь между высотой и радиусом кластеров на поздней стадии фазового перехода.

Равновесная толщина пленки $h_{\min }^{*}$ вне наночастиц, сформированных вдали от границы метастабильности $h_{s}^{*}$, уменьшается по мере роста их размера (см. рис. $3, b$ ). Наличие данной убывающей зависимости равновесной толщины пленки $h_{\min }^{*}$ от размера является необходимым условием для реализации механизма коалесценции на поздней стадии фазового перехода.

\section{4. Ранняя стадия формирования кластеров}

Рассмотрим раннюю стадию трансформации тонкой пленки, характеризуемой средней толщиной $\bar{h}^{*}$, соответствующей области нестабильных состояний $\left(\bar{h}^{*} \geq h_{s}^{*}\right)$, и эффективным потенциалом взаимодействия с подложкой (6). Пусть в начальный момент времени кластеры на поверхности отсутствовали, а толщина пленки характеризовалась малыми флуктуациям. Для данной системы следует ожидать образования протяженных участков с малой толщиной, близкой к равновесному значению $\left(h^{*} \sim h_{e q}^{*}\right)$. При этом перераспределенная на поверхности жидкость приводит к формированию уединенных кластеров [3-6,15-18]. Проведем анализ кинетики начальной стадии трансформации данной пленки.

Для выполнения данной задачи было проведено численное решение дифференциального уравнения (5) с эффективным потенциалом (6) и периодическими граничными условиями. Решение уравнения проводилось с помощью спектрального метода [20,21,31]. Размер области моделирования для всех рассмотренных ниже случаев принимался равным $S=1024 l \times 1024 l$. Основными критериями выбора параметров моделирования являются устойчивость и точность используемого численного метода, а также возможность наблюдения достаточного количества структурных элементов на поверхности пленки. В данном разделе параметр $\sigma_{0}$ был принят равным $\sigma_{0}=0.4$, шаг по времени составил $\Delta \tau=2.5 \cdot 10^{-4}$.

Результаты моделирования ранней стадии фазового перехода смачивания для случая тонкой пленки со средней толщиной $\bar{h}^{*}=2.5$ приведены на рис. 4, $a-d$. На рисунках показана область подложки размером $512 l \times 512 l$, то есть одна четвертая часть всей рассматриваемой системы. Из рисунков следует, что на начальной стадии формируются участки, которые имеют вид уединенных лунок и характеризуются значением толщины пленки $h^{*} \sim h_{e q}^{*}$ (рис. $\left.4, a\right)$. Размеры лунок и их концентрация постепенно увеличиваются (рис. $4, b)$, что приводит к их слиянию между собой (рис. 4,c). В результате слияния образуются сплошные протяженные участки, характеризуемые значением толщины $h^{*} \sim h_{e q}^{*}$. Данный процесс завершается объединением всех существующих тонких участков в один, в результате чего на поверхности формируются уединенные, несвязанные между собой островки (кластеры), часть из которых имеет сильно ассиметричную форму (см. рис. $4, d$ ). Рельеф поверхности оказывается сходным с распределением фаз, формирующимся в процессе спинодального распада в бинарных системах (см. например, [31]). Затем, образовавшиеся островки трансформируются так, чтобы достигнуть минимального значения энергии, которое 

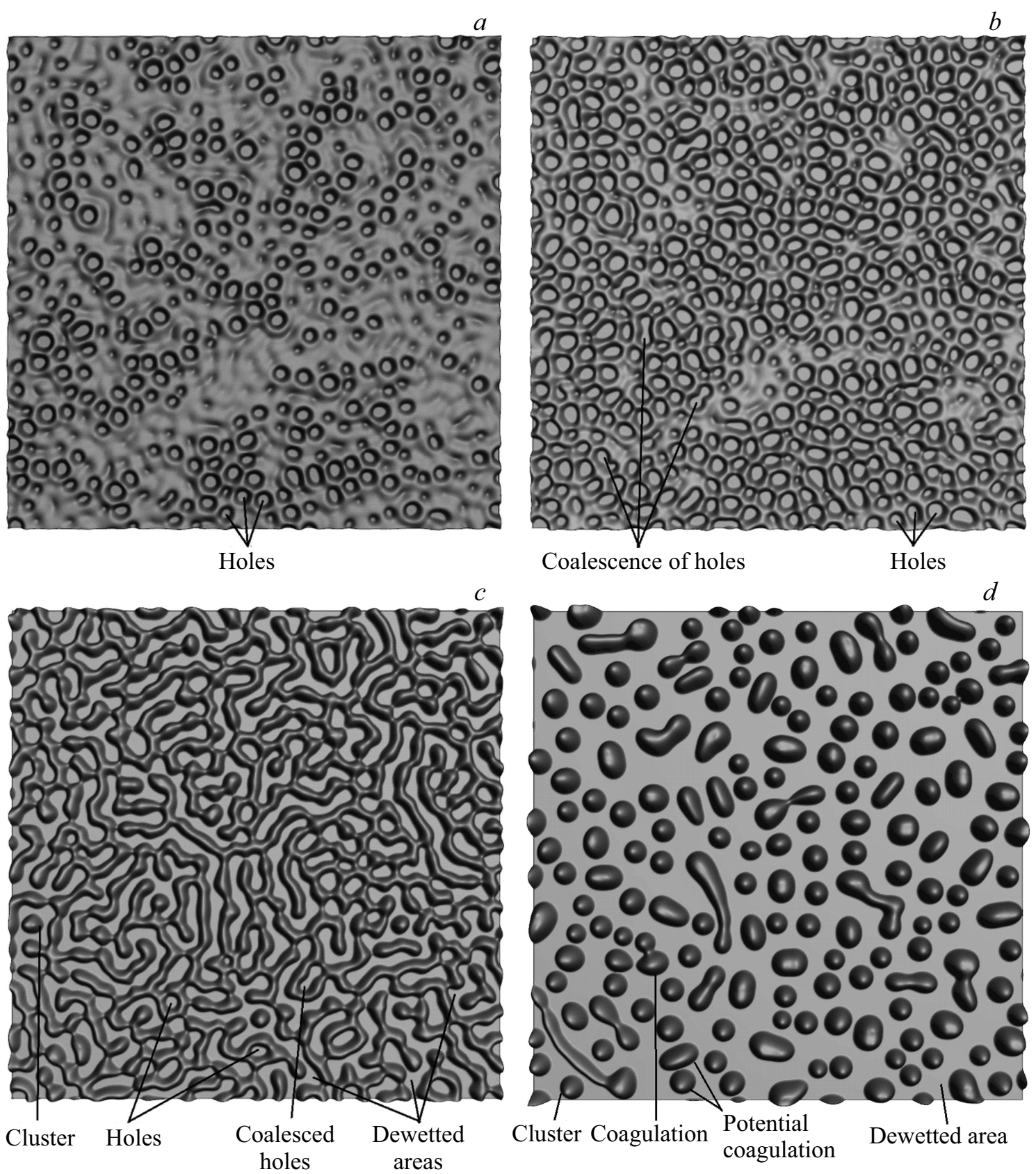

Coalescence of holes

Holes

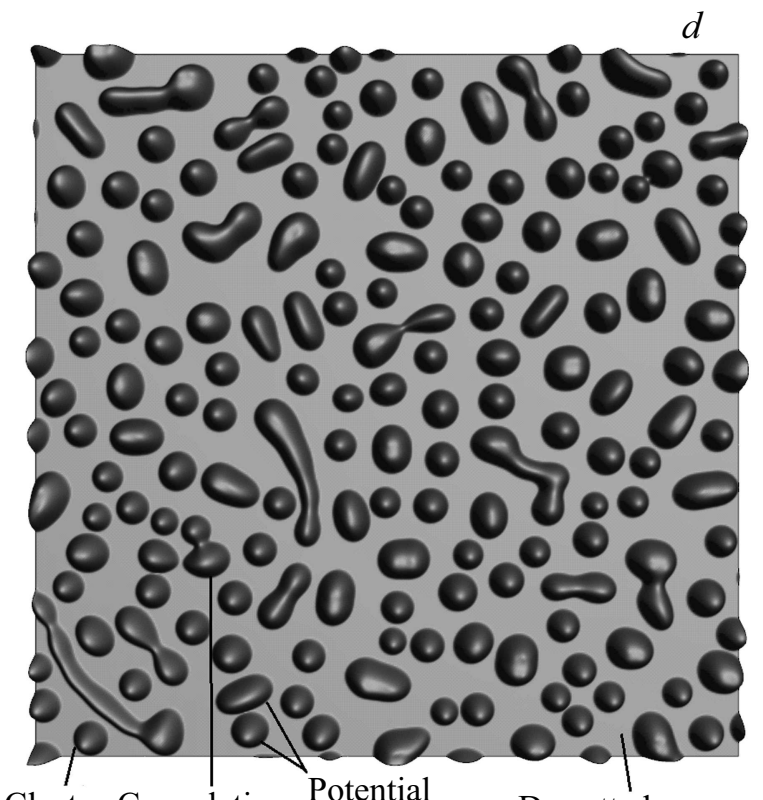

Cluster Coagulation Potential

coagulation

Dewetted area

Рис. 4. Результат расчета распределения толщины пленки $h^{*}(x, y)$ в различные моменты времени на ранней стадии для пленки характеризуемой средней толщиной $\bar{h}^{*}=3.0$ и $\sigma_{0}=0.4: a-\tau=122.5, b-\tau=142.5, c-\tau=202.5, d-\tau=1252.5$. Приведенные результаты расчета рельефа пленки соответствуют фрагменту поверхности с размером $512 l \times 512 l$.

обеспечивается, если кластеры соответствуют круговой симметрии (рис. $4, d)$. Часть кластеров в процессе такой трансформации взаимодействуют между собой путем их слияния (коагуляции). Образовавшиеся в результате слияния кластеры также стремятся к состоянию с круговой симметрией.

Общий характер эволюции пленки на ранней стадии эволюции согласуется с результатами других авторов [3-6,15-17], которые могут быть существенно дополнены выводами о кинетике некоторых характеристик рассматриваемого ансамбля структурных единиц (лунок), таких как средний размер, концентрация и функция распределения по размерам.

Идентификация отдельных лунок, образовавшихся на поверхности пленки, в каждый момент времени проводилась на основе метода ближайшего соседа [20,32,33]. В качестве линейного размера лунки принимался эффективный радиус ее проекции на плоскость подложки

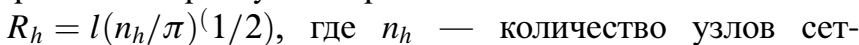
ки, соответствующих рассматриваемой лунке $\left(h^{*}<h_{c}^{*}\right)$. Значения порогового значения толщины пленки $h_{c}^{*}$ принималось равным $h_{c}^{*}=1.5$. Концентрация лунок $X_{h}$ 

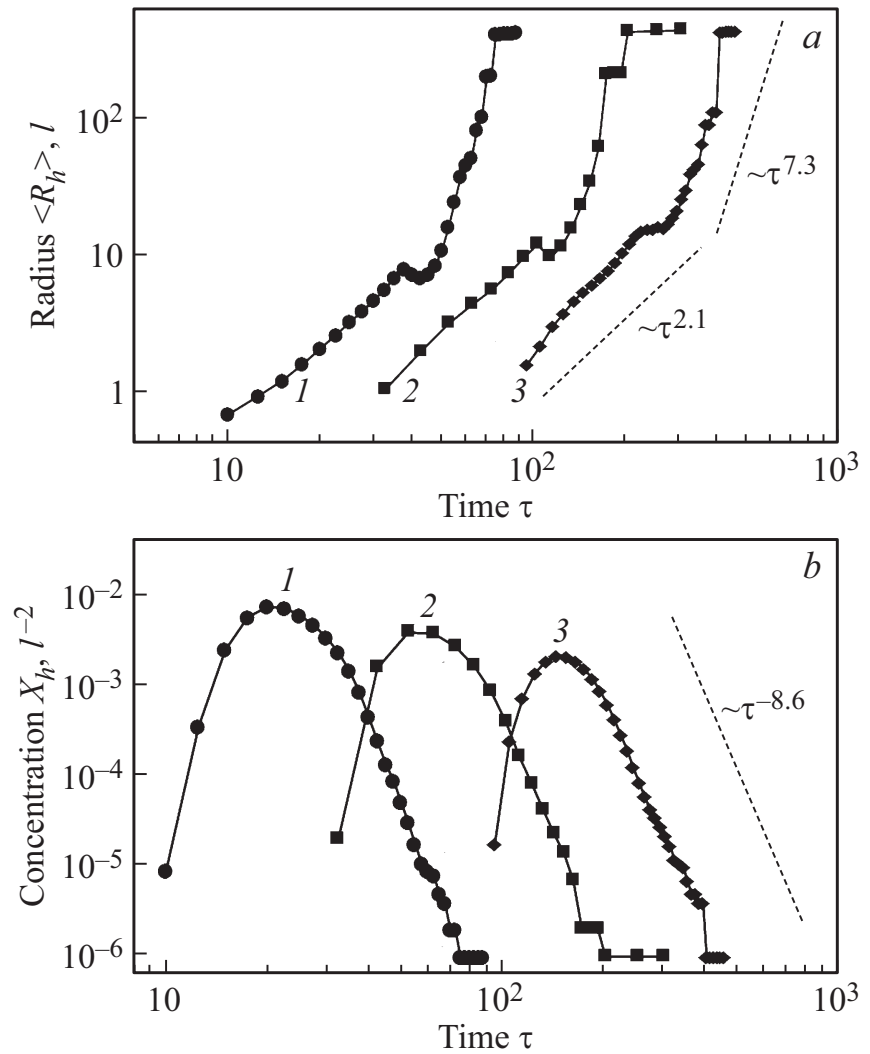

Рис. 5. Кинетика концентрации лунок (рис. $a$ ) и их среднего размера (рис. $b$ ) на ранней стадии смачивающего фазового перехода в пленке, характеризуемой различными значениями исходной толщины: $1-\bar{h}^{*}=2.0,2-\bar{h}^{*}=2.5,3-\bar{h}^{*}=3.0$.

рассчитывалась как $X_{h}=N_{h} / S$, где $N_{h}$ - количество лунок, обнаруженных в рассматриваемой области моделирования $S$.

Кинетика зарождения и роста лунок приведена на рис. 5, где показана расчетная зависимость их среднего радиуса (рис. $5, a)$ и концентрации (рис. $5, b)$ от времени. В начальный момент времени лунки полностью отсутствуют, их возникновение происходит после некоторого инкубационного периода (рис. $5, a, b)$. Аналогично росту выделений вторых фаз в твердых растворах лунки проходят стадии зарождения и роста (рис. $5, a, b)$. Следует заметить, что на стадии роста лунок, когда их концентрация изменяется достаточно слабо, средний радиус удовлетворительно описывается степенной зависимостью $R_{\bar{h}} \propto \tau^{2.1}$. Поскольку концентрация лунок достаточно велика, то основным механизмом взаимодействия между ними является их слияние. На стадии слияния средний размер демонстрирует быстрый, лавинообразный рост, который может быть приблизительно описан в виде степенных зависимостей среднего радиуса $R_{h} \propto \tau^{7.3}$ и концентрации $X_{h} \propto \tau^{-8.6}$. Процесс слияния лунок на поверхности подложки формально завершается при формировании одного сплошного участка, характеризуемого толщиной $h^{*} \sim h_{e q}^{*}$, что отражается постоянными значениями $R_{h}$ и $X_{h}$ на рис. 5. Различие показателей степенных зависимостей для рассмотренных толщин пленок невелико и не превышает $\sim 10 \%$. При завершении слияния лунок (рис. $4, d, 5)$ формируются устойчивые уединенные островки (кластеры), разделенные между собой протяженными участками с толщиной пленки $h^{*} \sim h_{e q}^{*}$.

\section{5. Кинетика образования кластеров на поверхности тонких пленок}

Для анализа формирования кластеров на поверхности подложки было проведено моделирование для трех тонких пленок, имеющих толщину $\bar{h}^{*}$, равную $2.0,2.5,3.0$ при $\sigma_{0}=0.2$ и $\Delta \tau=2.5 \cdot 10^{-4}$. Результаты моделирования эволюции кластеров для случая пленки, имеющей толщину $\bar{h}^{*}=2.0$, представлены на рис. 6. На рисунках отображены участки, имеющие размер $256 l \times 256 l$, то есть одна шестнадцатая от всей рассматриваемой площади подложки $S$. Предшествующая стадия формирования лунок и их слияния аналогична рис. 4, $a-d$. На разных стадиях формирования кластеров для рассматриваемых фрагментов пленок площадью $S$ наблюдалось $10^{2}-10^{4}$ кластеров, что является достаточным для анализа рассматриваемых средних характеристик и функции распределения по размерам.

Из рисунка следует, что после завершения слияния лунок (рис. 4, $d$ и 6, $a$ ) происходит постепенное перераспределение вещества между сформировавшимися кластерами, которые могут изменяться как по своему размеру, так и по высоте. В процессе трансформации пленки могут быть реализованы механизмы, сходные с процессом фазового перехода первого рода в бинарных сплавах. Наблюдается некоторый рост размера кластеров за счет поглощения оставшегося вещества из пленки, где $h^{*}>h_{e q}^{*}$. Близлежащие островки могут объединяться в более крупные за счет латерального взаимодействия (рис. 6, $b$ и $c$ ), то есть происходит их коагуляция (слияние). На поздней стадии происходит перенос вещества от кластеров малого размера к более крупным (см. рис. 6, с и $d$ ), что связано с убыванием равновесной толщины пленки при увеличении размера кластеров (см. рис. $3, b$ ). При этом рост крупных кластеров обеспечивается за счет исчезновения мелких, то есть реализуется механизм коалесценции. Корреляция между высотой и размером наблюдаемых кластеров (рис. 6) оказывается достаточно близкой к аналогичной зависимости для равновесных кластеров, представленной на рис. $3, a$.

Необходимым является установление закономерностей кинетики изменения среднего эффективного радиуса, средней высоты и концентрации сформированных кластеров. Идентификацию кластеров на поверхности подложки будем проводить на основе метода ближайшего соседа аналогично рассмотренной в предыдущем разделе процедуре идентификации лунок $[20,31,32]$. При этом к участкам, соответствующим кластерам, будем относить узлы, удовлетворяющие условию $h^{*} \geq h_{c}^{*}$, 

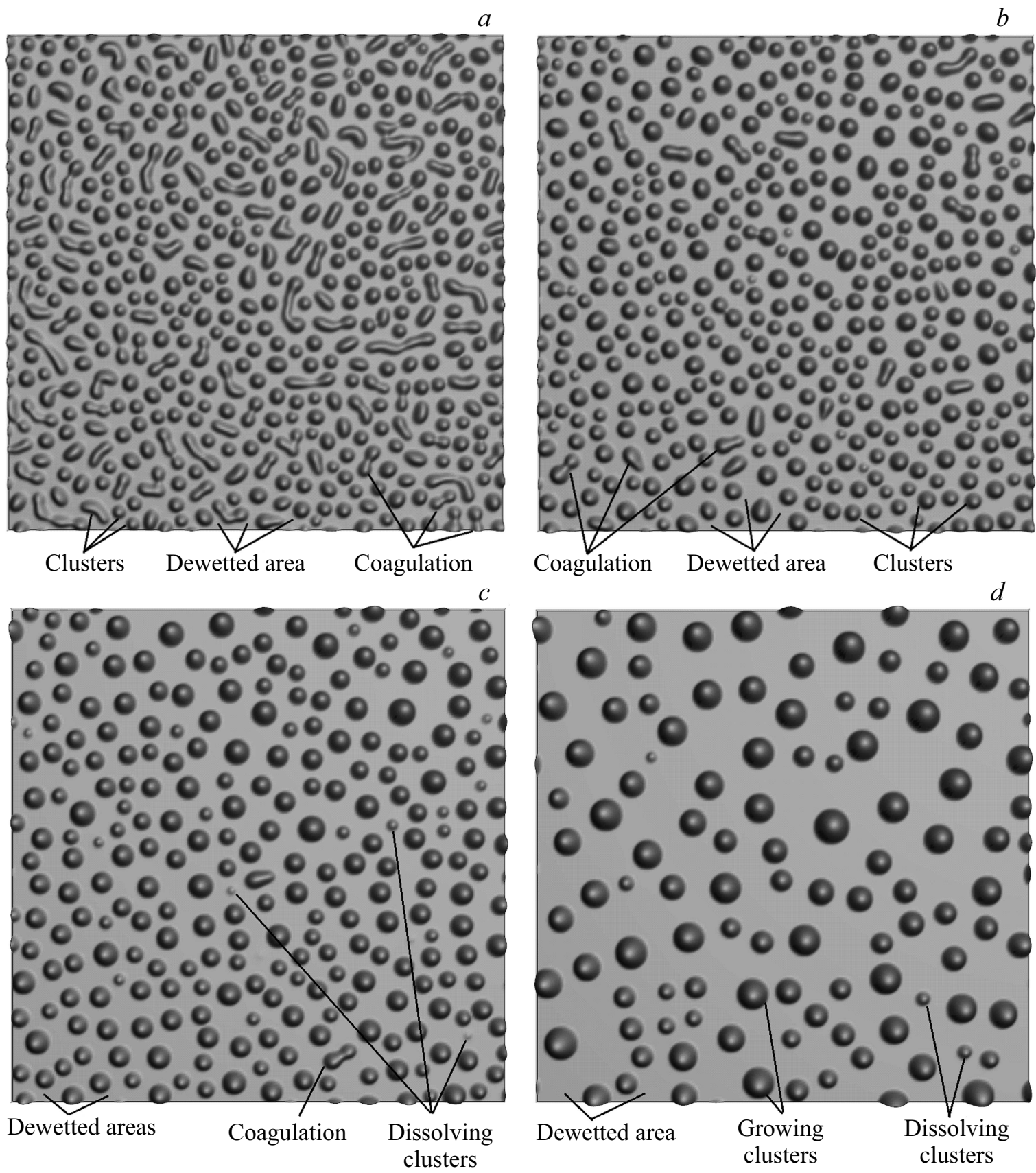

Рис. 6. Результат моделирования роста кластеров на поверхности подложки в процессе смачивающего фазового перехода для пленки $\left(\bar{h}^{*}=2.0, \sigma_{0}=0.2\right): a-\tau=42, b-\tau=82, c-\tau=442, d-\tau=3002$. Приведенные результаты расчета рельефа пленки соответствуют фрагменту поверхности с размером $256 l \times 256 l$.

$\left(h_{c}^{*}=1.5\right)$. В качестве характеристики высоты кластера примем величину $H^{*}$, равную максимальному значению толщины пленки $H^{*}=\max h^{*}$ в рассматриваемом кластере. Размер кластера, так же как и размер лунок, будем характеризовать эффективным радиусом $R_{C}=l\left(n_{C} / \pi\right)^{1 / 2}$, где $n_{C}-$ количество узлов сетки, соответствующих выбранному кластеру. Концентрация кластеров на поверхности подложки равна $X_{C}=N_{C} / S$, где $N_{C}$ - количество обнаруженных кластеров на подложке $S$.
Результаты расчета среднего размера $\left\langle R_{C}\right\rangle$, средней высоты $\left\langle H^{*}\right\rangle$ и концентрации $X_{C}$ кластеров приведены на рис. 7. Постоянное значение среднего радиуса (рис. 7, $a$ ) и концентрации (рис. 7,b) на малых временах соответствует сплошной пленке, сформированной в качестве начального условия, для которой как кластеры, так и лунки отсутствуют, а средняя толщина превышает выбранное пороговое значение $\bar{h}^{*}>h_{c}$. Следующее за данным участком скачкообразное изменение данных характеристик (рис. 7, $a-c$ ) соответствует лавинообраз- 

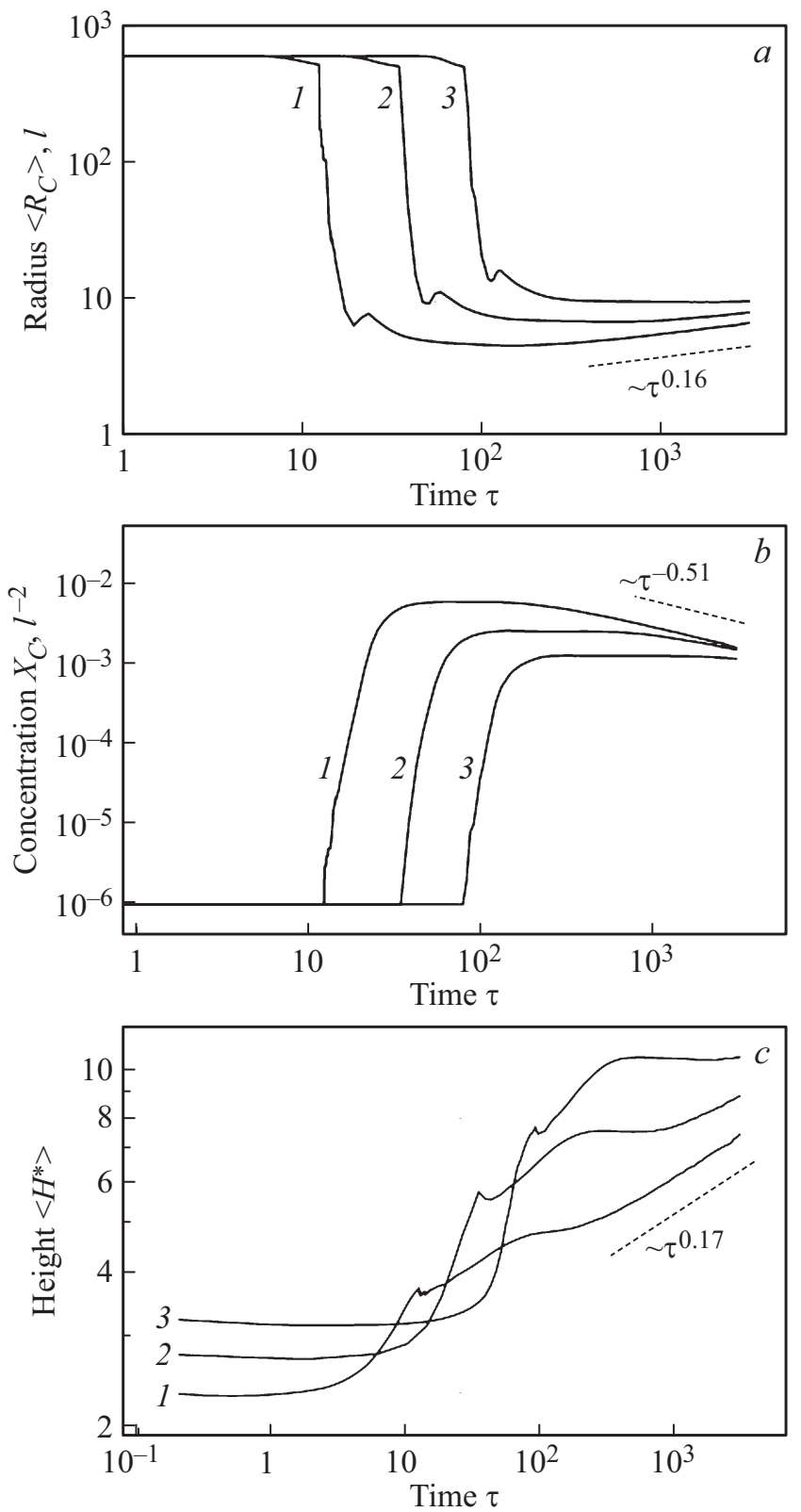

Рис. 7. Результаты расчета кинетики формирования кластеров на поверхности пленки в процессе смачивающего фазового перехода: $a-$ средний радиус $\left\langle R_{C}\right\rangle, b-$ концентрация $X_{C}$, $c$ - высота $\left\langle H^{*}\right\rangle$. Расчет проведен для параметра $\sigma_{0}=0.2$. Кривые соответствуют различной начальной толщине пленки: $1-\bar{h}^{*}=2.0,2-\bar{h}^{*}=2.5,3-\bar{h}^{*}=3.0$.

ному слиянию лунок между собой (см. рис. $4, b-d$ и 5). При этом происходит изменение эффективного коэффициента диффузии, который определяется второй производной потенциала $V^{*^{\prime \prime}}$ и становится положительным в области между образующимися кластерами $\left(h^{*}<h_{s}^{*}\right)$. На следующем этапе происходит трансформация кластеров, связанная с уменьшением их среднего размера $\left\langle R_{C}\right\rangle$ и увеличением средней высоты $\left\langle H^{*}\right\rangle$, при этом концентрация $X_{C}$ практически не изменяется. Данный интервал соответствует переходу кластеров от неправильной формы (см. рис. 4, d,6a) к круговой симметрии (см. рис. 6, $b$ ). На поздней стадии формирования пленки наблюдается снижение концентрации кластеров $X_{C} \propto \tau^{-0.51}$, которое сопровождается ростом их среднего радиуса $\left\langle R_{C}\right\rangle \propto \tau^{0.16}$ и высоты $\left\langle H^{*}\right\rangle \propto \tau^{0.17}$. Среднее значение избыточной толщины пленки между кластерами также убывает по степенному закону, близкому к: $\left(h^{*}-h_{e q}^{*}\right) \propto \tau^{-0.23}$. Поскольку средняя толщина пленки между кластерами приближается к постоянному равновесному значению $h_{e q}^{*}$, и выполняется закон сохранения вещества в пленке, то на поздней стадии можно ожидать постоянства выражения $X_{C}\left\langle H^{*}\right\rangle^{2}\left\langle R_{C}\right\rangle^{2} \approx$ const, что удовлетворительно выполняется.

Полученные значения асимптотических решений отличаются от полученных зависимостей в работах $[15,16]$, где для одномерного случая и потенциала (6) при $m=3$ и $n=2$ на основе анализа кинетических уравнений установлено, что $X_{C} \propto \tau^{-2 / 5}$ и $\left\langle R_{C}\right\rangle \propto \tau^{1 / 5}$, зависимости избыточной толщины между кластерами и зависимость средней высоты кластеров не рассматривались. Отличие полученных результатов от работ $[15,16]$ может быть связано с отличием размерности рассмотренной задачи, а также возможной незавершенностью перехода к асимптотической стадии.

Для оценки точности расчетов было проведено сравнение результатов решения уравнения (5) с использованием выбранного спектрального метода для двух значений шага по времени $\Delta \tau$ и $\Delta \tau / 2$ (см. например, [21]) и одинаковыми начальными условиями. Сравнение результатов расчета кинетики среднего размера, концентрации и средней высоты островков для трех рассматриваемых пленок на интервале $0<\tau<1200$ приводит к оценке относительной ошибки расчета, которая для всех рассматриваемых величин не превышает $0.3 \%$.

Выполненное моделирование позволяет также проследить за эволюцией функции распределения кластеров как по эффективному радиусу $R_{C}$, так и по высоте $H^{*}$. Результаты расчета приведены на рис. 8. На ранней стадии эволюции кластеров функция распределения по эффективному радиусу (рис. $8, a$ ) существенно вытянута в сторону больших размеров, что обусловлено как сильной асимметрией образовавшихся на ранней стадии кластеров (рис. 4, $d, 6, a$ ), так и слиянием кластеров друг с другом (рис. 4, $d, 6, a$ и $b$ ). На этой стадии размер кластеров может более чем в два раза превышать соответствующее среднее значение. На стадии роста за счет поглощения вещества из пространства между кластерами функция распределения оказывается практически симметричной (рис. $8, b$ ). Затем, по мере перехода к механизму коалесценции, функция распределения по размерам снова становится несимметричной, вытянутой в сторону малых размеров (рис. 8,c), то есть приобретает вид, сходный с равновесной функцией распределения в теории коалесценции островков на поверхности подложки [25]. Динамика функции распределения кластеров по высоте приведена на рис. 8, $d-e$. На ранней стадии формирования все кластеры имеют 

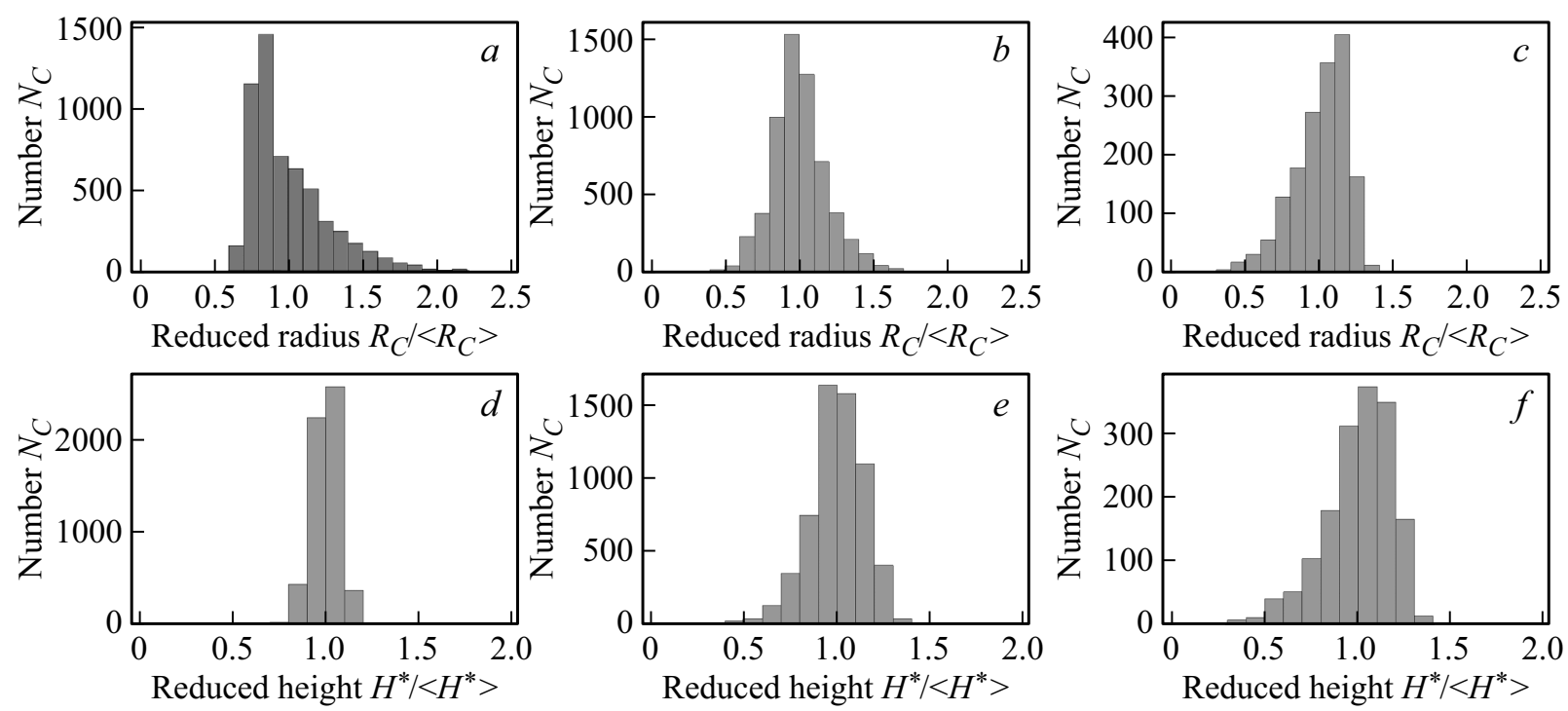

Рис. 8. Функции распределения островков по размеру (рис. $\boldsymbol{a}-\boldsymbol{c}$ ) и высоте (рис. $d-e$ ) в процессе смачивающего фазового перехода пленки $\left(\bar{h}^{*}=2.0, \sigma_{0}=0.2\right)$ в различные моменты времени $\tau: a, d-\tau=42, b, e-\tau=102, c, f-\tau=3002$.

очень близкое значение высоты $H^{*}$ (рис. $8, d$ ), в отличие от сильно несимметричной функции распределения по радиусу (рис. 8, $a$ ). На поздней стадии функция распределения кластеров по высоте (рис. 8,e,f) описывается сходной динамикой с распределением по радиусу. Данный результат согласуется с выводами теории эволюции дисперсионных систем на поверхности твердых тел [25], в которой для стадии коалесценции установлено подобие функций распределения по высоте и по радиусу для кластеров, имеющих цилиндрическую геометрию.

\section{6. Заключение}

В работе на основе гидродинамической модели исследован процесс трансформации тонких пленок жидкости на поверхности подложки, эффективный потенциал взаимодействия между атомами которых характеризуется потенциалом Леннард-Джонса.

Установлены закономерности формирования равновесных кластеров на поверхности подложки, имеющих круговую симметрию. Показано, что для рассмотренного потенциала взаимодействия высота крупных равновесных кластеров линейно зависит от его размера.

На ранней стадии трансформации пленки основным механизмом является зарождение лунок, их рост и последующее лавинообразное слияние. Данный процесс завершается образованием уединенных кластеров на поверхности пленки, разделенных протяженными участками с толщиной пленки, близкой к равновесной. На ранней стадии формирования лунок кинетика их среднего размера удовлетворительно описывается степенной зависимостью от времени $\left\langle R_{h}\right\rangle \propto \tau^{2.1}$. На стадии слияния средний размер лунок может быть аппроксимирован заметно более быстрой степенной зависимостью $\left\langle R_{h}\right\rangle \propto \tau^{7.3}$, при этом их концентрация убывает как
$X_{h} \propto \tau^{-8.6}$. Функция распределения лунок по размерам на поздней стадии оказывается существенно вытянутой в область больших размеров, что соответствует наблюдаемому механизму слияния.

В процессе лавинообразного слияния лунок образуются протяженные участки малой толщины, в которых одновременно формируются кластеры. Данные кластеры проходят стадии зарождения, роста и коалесценции. В процессе эволюции ансамбля кластеров на поверхности подложки изменяется их эквивалентный радиус и высота. На начальном этапе эволюции кластеров наблюдается рост их высоты за счет уменьшения радиуса, то есть происходит стягивание пленки. При этом кластеры продолжают поглощение вещества из пленки между кластерами. На стадии коалесценции происходит слабый степенной рост среднего размера кластеров и их средней высоты $\left(\left\langle R_{c}\right\rangle \propto \tau^{0.16},\right\rangle H^{*}\left\langle\propto \tau^{0.17}\right)$. Данные величины увеличиваются практически прямопропорционально друг другу, что соответствует сделанным выше выводам о линейном изменении высоты равновесного кластера в зависимости от его радиуса. Концентрация кластеров на стадии коалесценции убывает $X_{C} \propto \tau^{-0.51}$. На данной стадии функции распределения по радиусу и по высоте оказываются подобными друг другу, что согласуется с выводами теории эволюции дисперсионных систем на поверхности твердых тел.

\section{Финансирование работы}

Работа выполнена при финансовой поддержке Министерства науки и высшего образования (проект № 00042019-0001), Российского фонда фундаментальных исследований и Правительства Ульяновской области (проект № 18-42-732002). 


\section{Конфликт интересов}

Авторы заявляют, что у них нет конфликта интересов.

\section{Список литературы}

[1] Nanoscale liquid interfaces / Ed. T. Ondarçuhu, J.P. Aimé. CRC Press Taylor \& Francis Group, Boca Raton (2013). $769 \mathrm{p}$.

[2] J. Cahn. J. Chem. Phys. 66, 3667 (1977).

[3] D. Bonn, J. Eggers, J. Indekeu, J. Meunier, E. Rolley. Rev. Mod. Phys. 81, 739 (2009).

[4] A. Oron, S.H. Davis, S.G. Bankoff. Rev. Mod. Phys. 69, 931 (1997).

[5] J. Becker, G. Grün, R. Seemann, H. Mantz, Kh. Jacobs, K.R. Mecke, R. Blossey. Nature Mater. 2, 59 (2003).

[6] R.V. Craster, O.K. Matar. Rev. Mod. Phys., 81, 1131 (2009).

[7] X.-J. Cai, J. Genzer, R.J. Spontak. Langmuir 30, 11689 (2014).

[8] M. Kalloudis, E. Glynos, S. Pispas, J. Walker, V. Koutsos. Langmuir 29, 2339 (2013).

[9] Я.Е. Гегузин, Ю.С. Кагановский. Диффузионные процессы на поверхности кристалла. Энергоатомиздат, М (1984), $128 \mathrm{c}$.

[10] Я.Е. Гегузин, Ю.С. Кагановский. УФН 125, 489 (1978).

[11] Б.Б. Страумал. Фазовые переходы на границах зерен. Наука, М. (2003), 327 с.

[12] С.В. Булярский. ЖТФ 81, 64 (2011).

[13] С.В. Булярский, А.С. Басаев. ФТТ 57, 1041 (2015).

[14] В.М. Самсонов, Н.Ю. Сдобняков, М.В. Самсонов, Д.Н. Соколов, Н.В. Новожилов. Поверхность. Рентгеновские, синхротронные и нейтронные исследования 8, 76 (2015).

[15] K.B. Glasner, T.P. Witelski. Phys. Rev. E 67, 16302 (2003).

[16] M.B. Graton, T.P. Witelski. Physica D 238, 2380 (2009).

[17] A.A. Pahlavan, L. Cueto-Felgueroso, A.E. Hosoi, G.H. McKinely, R. Juanes. J. Fluid Mech. 845, 642 (2018).

[18] K. Mahdy, A. Afkhami, L. Kondic. Phys. Fluids 26, 62002 (2016).

[19] Б.В. Дерягин, Н.В. Чураев, М.В. Муллер. Поверхностные силы. Наука, М. (1985) 398 с.

[20] P.E. L'vov, V.V. Svetukhin. Mod. Simul. Mater. Sci. Eng. 25, 075006 (2017).

[21] S. Dai, Q. Du. J. Comp. Phys. 310, 85 (2016).

[22] G. Grün, K. Mecke, M. Rauscher. J. Status Phys. 122, 1261 (2006).

[23] С.А. Кукушкин. Поверхность: Физика, химия, механика 7, $22(1983)$

[24] T.V. Sakalo, S.A. Kukushkin. Acta Met. Mater. 42, 2803 (1994).

[25] С.А. Кукушкин, В.В. Слезов. Дисперсные системы на поверхности твердых тел: механизмы образования тонких пленок (эволюционный подход). Наука, СПб. (1996). $304 \mathrm{c}$.

[26] R. Seeman, S. Herminghaus, K. Jacobs. Phys. Rev. Lett. 86, 5534 (2001).

[27] J.W. Cahn, J.E. Hilliard. J. Chem. Phys. 31, 688 (1959).

[28] H. Aaronson, M. Enomoto, J. Lee. Mechanisms of Diffusional Phase Transformations in Metals and Alloys. CRC Press, Taylor \& Francis Group, Boca Raton. (2010), 667 p.

[29] П.Е. Львов, В.В. Светухин. ФТТ 57, 1192 (2015).
[30] P.E. L’vov, V.V. Svetukhin. Mod. Simul. Mater. Sci. Eng. 27, 025002 (2019).

[31] J. Zhu, L.-Q. Chen. J. Shen, V. Tikare. Phys. Rev. E 60, 3564 (1999).

[32] M. Miller, R. Forbes. Atom-probe tomography: the local electrode atom probe. Springer, N. Y. (2014) 423 p.

[33] П.Е. Львов, В.В. Светухин. Св-во о гос. регистрации программы для ЭВМ. № 2017616554 от 08.07.2017.

Редактор Т.Н. Василевская 